\title{
Aleitamento materno e a prevenção da doença alérgica: uma revisão baseada na evidência
}

Marisa Monteiro Gomes, ${ }^{1}$ Susana Patricia Leal Rebelo ${ }^{2}$

\section{RESUMO}

Introdução: As doenças alérgicas estão entre as patologias mais comuns que afetam as crianças, tendo um impacto considerável na qualidade de vida dos doentes e/ou cuidadores. O leite materno é o alimento de eleição no primeiro ano de vida, com vários benefícios demonstrados. Contudo, o seu papel na prevenção de doenças alérgicas é controverso.

Objetivo: Comparar o efeito do aleitamento materno (AM) com o do leite adaptado ou misto na redução da doença alérgica nas crianças até aos 18 anos de idade.

Fonte de dados: Base de dados MEDLINE e sítios eletrónicos de medicina baseada na evidência.

Métodos de revisão: Pesquisa de estudos publicados entre janeiro de 2007 e março de 2017, em português, inglês e espanhol, utilizando os termos MeSH breast feeding, atopic dermatitis, allergic rhinitis, asthma e food hypersensivity. Para avaliação da qualidade dos estudos e força de recomendação foi utilizada a escala Strength of Recommendation Taxonomy, da American Family Physician (SORT).

Resultados: Da pesquisa obtiveram-se 307 artigos, dos quais sete preencheram os critérios de inclusão: uma meta-análise, uma revisão sistemática, dois ensaios clínicos aleatorizados, um estudo observacional e duas normas de orientação clínica. Globalmente, o AM parece ter um efeito protetor relativamente ao aparecimento de dermatite atópica até aos dois anos de idade, apesar de alguns estudos não demonstrarem qualquer associação. Em relação à rinite alérgica, o AM foi protetor até aos cinco anos de idade e na asma até aos 18 anos. Por fim, para alergia alimentar não foi encontrada associação com o AM.

Conclusão: O AM tem efeito protetor no aparecimento de asma (SORT A) e rinite alérgica (SORT B). Relativamente à alergia alimentar e à dermatite atópica, os resultados são controversos. Assim, não é possível confirmar o seu efeito protetor (SORT B). São, portanto, necessários mais estudos de boa qualidade e por períodos mais alargados.

Palavras-chave: Aleitamento materno; Doença alérgica; Asma; Rinite alérgica; Dermatite atópica.

\section{INTRODUÇÃO}

A s doenças alérgicas afetam a qualidade de vida de milhões de crianças e adultos, sendo, além disso, responsáveis por gastos consideráveis em recursos sociais e económicos, ${ }^{1}$ o que as torna um importante problema de saúde pública.

A sua prevalência tem vindo a aumentar nas últimas décadas, ${ }^{2}$ pelo que é premente a descoberta dos fatores responsáveis por este crescimento, tendo os fatores

1. Interna de Medicina Geral e Familiar, USF Antonina, ACeS Ave Famalicão. 2. Médica Assistente de Medicina Geral e Familiar. UCSP Lustosa, ACeS Vale Sousa Norte. ambientais, nomeadamente os nutricionais, sido amplamente estudados.

O leite materno (LM) é o alimento de eleição recomendado no primeiro ano de vida, com efeito benéfico demonstrado a nível do desenvolvimento físico e psicológico do lactente. ${ }^{3} \mathrm{~A}$ ausência de aleitamento materno (AM) tem sido associada a diversas doenças na infância, nomeadamente doença celíaca, obesidade e doenças infeciosas (e.g., gastroenterite, infeções respiratórias superiores)..$^{3-4}$ Além disto, pensa-se que o AM tenha um papel protetor na prevenção das doenças alérgicas, embora a literatura não seja consensual. ${ }^{3}$ 
O objetivo primário deste estudo consiste em verificar o efeito do AM, comparativamente ao aleitamento artificial ou leite adaptado na redução da dermatite atópica, rinite alérgica, asma e alergia alimentar em crianças até aos 18 anos de idade.

\section{MÉTODOS}

Foi efetuada uma pesquisa de meta-análises (MA), revisões sistemáticas (RS), ensaios clínicos controlados e aleatorizados (ECA), estudos originais (EO) e normas de orientação clínica (NOC), nas bases de dados ME-

DLINE, Guidelines Finder, National Guideline Clearinghouse (NICE), Canadian MedicalAssociation Infobase, Canadian Task Force on Preventive Health, US Preventive Services Task Force, The Cochrane Library, DARE, Bandolier, TRIP e Índex das Revistas Médicas Portuguesas. Utilizaram-se os termos MeSH: breast feeding, atopic dermatitis, allergic rhinitis, asthma e food hypersensivity. Foram selecionados os artigos publicados entre 1 de janeiro de 2007 e 31 de março de 2017, em inglês, espanhol e português.

Foram critérios de inclusão:

- População: crianças até aos 18 anos de idade;

- Intervenção: aleitamento materno;

- Comparação: leite adaptado ou aleitamento misto;

- Outcomes: efeitos na doença alérgica, nomeadamente dermatite atópica, rinite alérgica, asma e alergia alimentar.

Foram excluídos: estudos com crianças pré-termo; estudos com suplementação materna ou infantil com nutrientes (e.g., ácidos gordos, prebióticos, probióticos); estudos com introdução de leite de vaca e estudos com exposição a alergénios específicos. Foram excluídos ainda artigos duplicados, artigos de opinião, artigos de revisão clássica do tema, ensaios clínicos incluídos nas RS ou MA selecionadas, resumos de sítios na Internet e artigos discordantes do objetivo da revisão.
A robustez metodológica dos ensaios clínicos incluídos foi avaliada utilizando a escala de Jadad. ${ }^{5}$ A qualidade dos estudos e a força de recomendação foram avaliadas utilizando a escala de Strength of Recommendation Taxonomy (SORT), da American Family Physician. ${ }^{6}$

\section{RESULTADOS}

Da pesquisa realizada obteve-se um total de 307 artigos. Destes, sete preencheram os critérios de inclusão: uma MA, uma RS, dois ECA, um EO e duas NOC. Os Quadros I e II resumem as características dos estudos selecionados para revisão. Os restantes foram excluídos por divergirem dos objetivos do trabalho, por não cumprirem os critérios de inclusão ou por serem repetidos. Também foram excluídos os ECA incluídos nas MA e RS.

De forma a facilitar a interpretação optou-se por analisar os resultados obtidos separadamente para cada um dos objetivos desta revisão: 1) o AM e o desenvolvimento da doença alérgica; 2) dermatite atópica; 3) rinite alérgica; 4) asma; 5) alergia alimentar.

\section{O aleitamento materno e o desenvolvimento de doença alérgica em geral}

O papel do AM na prevenção da doença alérgica tem sido alvo de estudo por diferentes autores, contudo com 


\section{QUADRO I. Meta-análise, revisão sistemática, ensaios clínicos e estudo observacional}

\section{Revisão sistemática}

\begin{tabular}{l|l|l}
\hline Referência & \multicolumn{1}{|c}{ População } & \multicolumn{1}{c}{ Intervenção } \\
\hline Kramer, et al. (2012) & Crianças até aos 18 anos de idade & $\begin{array}{l}\text { AM exclusivo >= 6 meses vs AM exclusivo durante 3-4 meses } \\
+ \text { aleitamento misto até aos } 6 \text { meses }\end{array}$ \\
\hline
\end{tabular}

Meta-análise

\begin{tabular}{l|l|l}
\hline Referência & \multicolumn{1}{|c}{ População } & \multicolumn{1}{c}{ Intervenção } \\
\hline Lodge, et al. (2015) & Crianças até aos 18 anos de idade & $\begin{array}{l}\text { Crianças sempre amamentadas vs crianças que nunca foram } \\
\text { amamentadas: } \\
\end{array}$ \\
& $\begin{array}{l}\text { AM }<3-4 \text { meses vs superior } \\
\text { AM prolongado vs curto }\end{array}$ \\
\hline
\end{tabular}

Ensaios clínicos

\begin{tabular}{l|l|l}
\hline Referência & \multicolumn{1}{|c|}{ População } & \multicolumn{1}{c}{ Intervenção } \\
\hline Kramer, et al. (2007) & $\begin{array}{l}\text { 17.046 pares mãe-criança, sendo que 13.889 } \\
\text { foram seguidos até aos 6,5 anos }\end{array}$ & Promoção do AM segundo o modelo da WHO/UNICEF \\
\hline Giwercman, et al. (2010) & $\begin{array}{l}\text { 411 recém-nascidos com um mês de idade } \\
\text { seguidos nos primeiros dois anos de vida }\end{array}$ & AM exclusivo \\
\hline
\end{tabular}

\section{Estudo observacional}

\begin{tabular}{l|l|l}
\hline Referência & \multicolumn{1}{|c|}{ População } & \multicolumn{1}{c}{ Intervenção } \\
\hline Hong, et al. (2014) & 10.383 crianças até aos 13 anos & AM exclusivo \\
\hline
\end{tabular}

Legenda: $\mathrm{AM}=$ aleitamento materno.

\section{QUADRO II. Normas de orientação}

\section{Normas de orientação clínica}

\begin{tabular}{l|l}
\hline Referência & \multicolumn{1}{c}{ Recomendações } \\
\hline EAACI - Food Allergy and Anaphylaxis Guidelines (2014) & $\begin{array}{l}\text { Recomenda o AM exclusivo para todas as crianças durante os primeiros } \\
\text { quatro a seis meses de vida }\end{array}$ \\
\hline NICE - Maternal and child nutrition (2014) & $\begin{array}{l}\text { Recomenda o AM exclusivo durante os primeiros seis meses de vida da } \\
\text { criança e durante a introdução de alimentos sólidos }\end{array}$ \\
\hline
\end{tabular}

Legenda: $\mathrm{AM}$ = aleitamento materno; $\mathrm{DA}$ = dermatite atópica.

resultados controversos. Enquanto alguns estudos demonstraram um papel protetor do AM nas doenças alérgicas, muitos outros não encontraram qualquer associação ou, pelo contrário, encontraram um aumento do risco de doença alérgica. ${ }^{7}$
Uma das principais razões pelas quais o efeito do AM no desenvolvimento de alergias permanece em discussão deve-se à complexidade imunológica do LM. Assim, para além da imunidade passiva através das imunoglobulinas A e G, o LM contém também outros 
fatores que estimulam o sistema imunitário infantil. Consequentemente, o LM fornece tanto sinais protetores como estimulatórios que podem conferir efeitos contraditórios no desenvolvimento do sistema imune e, consequentemente, levar ao desenvolvimento de suscetibilidade para a doença alérgica. ${ }^{7}$

Em 2014, a European Academy of Allergy and Clinical Immunology (EAACI) publicou uma norma de orientação clinica ${ }^{8}$ que recomenda o AM exclusivo para todas as crianças durante os primeiros quatro a seis meses de vida.

Os componentes imunomoduladores como, por exemplo, os ácidos gordos de cadeia longa e oligossacarídeos presentes no LM diferem de mãe para mãe, o que dificulta o estudo do efeito do LM na prevenção da

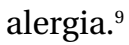

Também em 2014, o National Institute for Health and Care Excellence (NICE) publicou uma norma de orientação clinica ${ }^{4}$ que recomenda o AM durante os primeiros seis meses de vida da criança e enquanto for mutuamente desejado pela mãe e pelo filho. Refere ainda não haver evidência que suporte o aleitamento com fórmulas hidrolizadas para a prevenção de alergias quando comparado com o AM.

\section{Dermatite atópica}

A dermatite atópica é uma patologia frequente e com um importante impacto negativo na qualidade de vida. Nos primeiros meses de vida da criança, situações clínicas de infeções com repercussão cutânea podem ser erroneamente diagnosticadas como dermatite atópica e, desta forma, ser um viés quando se analisam os estudos.

Lodge e colaboradores ${ }^{7}$ encontraram pouca evidência do efeito protetor do AM na redução do risco de eczema até aos dois anos de idade, sendo que o efeito protetor se limitou ao AM exclusivo durante 3-4 meses em estudos transversais. Depois dos dois anos de idade, o efeito protetor do LM desapareceu e aumentou até o risco de dermatite atópica. O AM parece conferir alguma proteção em relação à dermatite atópica nas crianças de países menos desenvolvidos. Os autores explicam estes resultados com base na proteção contra infeções em idades precoces, nas dermatoses erroneamente diagnosticadas como dermatite atópica e ainda nas diferenças ambientais presentes nos países mais desenvolvidos que podem aumentar o risco da doença. Os estudos incluídos nesta meta-análise apresentavam baixa qualidade, tendo sido por isso atribuído um nível de evidência (NE) 2.

Kramer e colaboradores ${ }^{10}$ na sua RS incluíram dois estudos que compararam o efeito do AM exclusivo até aos 6-7 meses de idade versus o AM durante 3-4 meses e o eczema atópico nos primeiros 12 meses de vida. Concluíram que não existe redução significativa do risco de eczema atópico até ao ano de idade com o AM exclusivo.

No ECA de Kramer e colaboradores ${ }^{11}$ não ficou demonstrado um efeito protetor do AM exclusivo ou misto prolongado em relação ao eczema, nas crianças até aos 6,5 anos de idade. Contudo, os autores referem que o diagnóstico de dermatite atópica é controverso e que, como tal, pode estar subestimado.

À semelhança deste estudo, também Giwercman e colaboradores ${ }^{9}$ procuraram avaliar o efeito do AM na prevenção do eczema, verificando um aumento do risco de eczema com o AM exclusivo (RR=2,09; IC95\%, $1,15-3,08 ; p<0,05)$. Adicionalmente, foi encontrado um aumento do risco de eczema com o prolongamento da amamentação $(p<0,05)$.

O estudo observacional de Hong e colaboradores ${ }^{12}$ comparou a duração do AM e a associação com a dermatite atópica em crianças coreanas. Verificou-se que nas crianças com menos de cinco anos de idade, a prevalência da dermatite atópica foi 1,56 vezes superior com a amamentação prolongada (mais de 12 meses) quando comparada com o grupo de crianças não amamentadas (IC95\%, 1,26-1,94). Assim, a prevalência de dermatite atópica foi associada positivamente à duração da amamentação $(p<0,001)$. Em relação às crianças com mais de cinco anos de idade não foi encontrada nenhuma associação estatisticamente significativa entre a dermatite atópica e a duração da amamentação $(p=0,07)$. Por fim, o efeito da amamentação nesta doença não foi modificado pela história familiar de atopia. Os autores desta revisão explicam isto com base no facto de o leite materno conter muitos fatores, nomeadamente alergénios, toxinas e citocinas, que podem passar da mãe para a criança, assim como haver já uma predisposição genética destas crianças para o desenvolvimento de doenças alérgicas.

Em 2014, a NICE publicou uma norma de orientação 
clínica ${ }^{4}$ que recomenda o AM durante os primeiros seis meses de vida da criança e refere que o AM exclusivo durante os primeiros meses de vida pode reduzir o risco de dermatite atópica.

\section{Rinite alérgica}

Na meta-análise de Lodge e colaboradores, ${ }^{7}$ que incluiu cinco estudos coorte e 11 estudos transversais, verificou-se que o AM prolongado (superior a três meses) versus um curto período de tempo tem um efeito protetor na rinite alérgica, embora não significativo (OR=0,92; IC95\%, 0,84-1,01). Contudo, após a estratificação pela idade, este efeito protetor só foi encontrado nas crianças com menos de cinco anos de idade (OR=0,79; IC95\%, 0,63-0,98), não tendo sido encontrada nenhuma associação nas crianças depois dos cinco anos de idade (OR=1,05; IC95\%, 0,99-1,12). Um dos estudos transversais incluídos na MA estudou a interação com a história familiar de atopia e não encontrou associação.

\section{Asma}

Na meta-análise de Lodge e colaboradores, ${ }^{7}$ as crianças que foram amamentadas tiveram uma redução do risco de asma entre os cinco e os 18 anos de idade (OR=0,88; IC95\%, 0,82-0,95). Contudo, após a estratificação pelo nível de desenvolvimento do país, este efeito protetor foi superior nos países subdesenvolvidos.

Os autores explicam este facto com base na elevada prevalência de infeções respiratórias nas crianças destes países, sendo que estas são um importante fator de risco para o desenvolvimento de asma. Por outro lado, as medidas de higiene, vacinação e cuidados de saúde são também mais precários nestes países.

Quando estudado o AM exclusivo durante pelo menos três a quatro meses versus inferior a três meses nas crianças dos cinco aos 18 anos de idade não foi encontrada nenhuma associação entre o AM exclusivo superior a três meses e a redução de asma nestas crianças.

Pelo contrário, nessa mesma faixa etária, quando se comparou o AM prolongado (superior a três meses) versus um curto período de tempo este mostrou ser protetor quando prolongado (OR=0,90; IC95\%, 0,84-0,97) e, em especial, nos países menos desenvolvidos (OR=0,86; IC95\%, 0,79-0,94). Já quando se estratificou pela história familiar de atopia não foi encontrada ne- nhuma associação entre AM e asma nas crianças com e sem história familiar de atopia (OR=1,08; IC95\%, 0,74-1,58 e OR=1,2; IC95\%, 0,91-1,59, respetivamente).

A revisão sistemática de Kramer $^{10}$ verificou que não existe redução do risco de asma nas crianças entre os 5-6 anos de idade com o AM exclusivo.

Ressalve-se que as definições de asma nos diferentes estudos são variadas e que pode haver alguma confusão entre asma e sibilância recorrente. Por outro lado, houve diferenças em relação à faixa etária avaliada nos diferentes estudos.

\section{Alergia alimentar}

Um grande obstáculo nos estudos que avaliam a alergia alimentar é a sua própria definição.

Na meta-análise de Lodge e colaboradores ${ }^{7}$ não se encontrou associação entre o AM prolongado versus um curto período de tempo no desenvolvimento de AA $(\mathrm{OR}=1,02 ; \mathrm{IC} 95 \%, 0,88-1,18)$. Quando foi investigada a interação da história familiar de atopia com o AM e o desenvolvimento de alergia alimentar foi encontrado um risco superior nas crianças com história de atopia (OR=5,3; IC95\%, 1,2-24,1).

A revisão sistemática de Kramer $^{10}$ verificou que não existe redução do risco de alergia alimentar até ao ano de idade com o AM exclusivo até aos 6-7 meses de idade (RR=0,77; IC95\%, 0,25-2,41) nem aos cinco anos de idade (RR=0,61; IC95\%, 0,12-3,19).

\section{CONCLUSÃO}

$\mathrm{O}$ aleitamento materno tem benefícios para a mãe e para a criança, devendo por isso ser recomendado pelo menos nos primeiros quatro meses de vida e devendo ser continuado até aos seis meses de idade. ${ }^{13}$

Contudo, o seu papel na prevenção da doença alérgica permanece controverso.

Em relação ao efeito do AM na prevenção da dermatite atópica não existe evidência científica de que o AM seja protetor. Contudo, nas crianças de países menos desenvolvidos, parece que o AM confere alguma proteção em relação a esta patologia. Por outro lado, em alguns estudos houve inclusivamente uma associação positiva entre a dermatite atópica e o AM prolongado (duração superior a 12 meses).

No que concerne à rinite alérgica, o AM parece preveni-la em crianças até aos cinco anos de idade. Con- 
tudo, a prevalência desta patologia aumenta a partir dos três anos de idade, tendo um pico na adolescência; o facto de nos diferentes estudos não ter sido feito um acompanhamento das crianças até à adolescência pode ter levado à perda de alguns casos de rinite alérgica.

Relativamente à asma, os estudos foram mais consensuais, mostrando um efeito protetor do AM mesmo em crianças até aos 18 anos de idade.

Quanto à alergia alimentar, os estudos são mais limitados e o próprio diagnóstico desta patologia é controverso. Desta forma, não foi encontrada uma associação entre o AM e a prevenção da alergia alimentar.

A presente revisão apresenta várias limitações, a primeira das quais está relacionada com o facto dos estudos utilizados não serem uniformes na definição dos diferentes tipos de doenças alérgicas. Por outro lado, acresce ainda a multiplicidade de fatores externos envolvidos, nomeadamente o ambiente, a genética e a própria complexidade imunológica do LM e da sua interação com a flora intestinal e sistema imune do latente.

Outra limitação envolve o curto tempo de seguimento das crianças.

Assim, face ao exposto, é possível afirmar que o AM tem efeito protetor no aparecimento de asma (SORT A) e rinite alérgica (SORT B). Relativamente à alergia alimentar e à dermatite atópica, os resultados são controversos; como tal, não é possível confirmar o seu efeito protetor (SORT B). Desta forma, são necessários estudos de elevada qualidade, metodologia homogénea, amostras relevantes e mais prolongados para verificar o efeito do AM na doença alérgica ao longo do tempo.

\section{REFERÊNCIAS BIBLIOGRÁFICAS}

1. Ferreira $M$, Coelho R, Trindade JC. Prevenção primária da doença alérgica [Primary prevention of allergic disease]. Acta Med Port. 2007;20(3):215-9. Portuguese

2. Kliegman RM, Stanton BM, St Geme J, Schor N, Behrman RE, editors. Nelson's textbook of pediatrics. New York: Elsevier Health Sciences; 2011. ISBN 9781437735895

3. Schneider AP, Stein RT, Fritscher CC. O papel do aleitamento materno, da dieta e do estado nutricional no desenvolvimento de asma e atopia
[The role of breastfeeding, diet and nutritional status in the development of asthma and atopy]. J Bras Pneumol. 2007;33(4):454-62. Portuguese

4. National Institute for Health and Care Excellence. Maternal and child nutrition. London: NICE; 2014.

5. Jadad AR, Moore RA, Carroll D, Jenkinson C, Reynolds DJ, Gavaghan DJ, et al. Assessing the quality of reports of randomized clinical trials: is blinding necessary? Control Clin Trials. 1996;17(1):1-12.

6. Ebell MH, Siwek J, Weiss BD, Woolf SH, Susman J, Ewigman B, et al. Strength of recommendation taxonomy (SORT): a patient-centered approach to grading evidence in the medical literature. Am Fam Physician. 2004;69(3):548-56.

7. Lodge CJ, Tan DJ, Lau MX, Dai X, Tham R, Lowe AJ, et al. Breastfeeding and asthma and allergies: a systematic review and meta-analysis. Acta Paediatr. 2015;104(467):38-53.

8. Muraro A, Halken S, Arshad SH, Beyer K, Dubois AE, Du Toit G, et al. EAACI food allergy and anaphylaxis guidelines: primary prevention of food allergy. Allergy. 2014;69(5):590-601.

9. Giwercman C, Halkjaer LB, Jensen SM, Bønnelykke K, Lauritzen L, Bisgaard $\mathrm{H}$. Increased risk of eczema but reduced risk of early wheezy disorder from exclusive breast-feeding in high-risk infants. J Allergy Clin Immunol. 2010;125(4):866-71.

10. Kramer MS, Kakuma R. Optimal duration of exclusive breastfeeding: review. Cochrane Databse Syst Rev. 2012;(8):ID003517.

11. Kramer MS, Matush L, Vanilovich I, Platt R, Bogdanovich N, Sevkovskaya $Z$, et al. Effect of prolonged and exclusive breast feeding on risk of allergy and asthma: cluster randomised trial. BMJ. 2007;335(7624):815.

12. Hong S, Choi WJ, Kwon HJ, Cho YH, Yum HY, Son DK. Effect of prolonged breast-feeding on risk of atopic dermatitis in early childhood. Allergy Asthma Proc. 2014;35(1):66-70.

13. Mavroudi A, Xinias I. Dietary interventions for primary allergy prevention in infants. Hippokratia. 2011;15(3):216-22.

\section{CONFLITO DE INTERESSES}

Os autores declaram não ter quaisquer conflitos de interesse.

\section{FINANCIAMENTO DO ESTUDO}

Os autores não receberam qualquer financiamento para o desenvolvimento do estudo.

\section{ENDEREÇO PARA CORRESPONDÊNCIA}

Marisa Monteiro Gomes

E-mail: marisamonteirogomes@gmail.com

http://orcid.org/0000-0002-0427-1778

Recebido em 02-05-2017

Aceite para publicação em 08-01-2019 


\section{ABSTRACT}

\section{BREASTFEEDING AND ALLERGIC DISEASE PREVENTION: AN EVIDENCE-BASED REVIEW}

Introduction: Allergic diseases are among the most common diseases affecting children and have a substantial impact on the quality of life of patients and/or caregivers. Breast milk is the food of choice in the first year of life, with several established benefits. However, its role in the prevention of allergic diseases is controversial.

Objective: To determine the effect of breastfeeding compared with adapted formula or mixed feeding in the reduction of allergic disease in children up to 18 years of age.

Data sources: MEDLINE and Evidence-based medicine databases.

Review methods: Research of studies published between January 2007 and March 2017, in Portuguese, English, and Spanish, using the MeSH terms: breastfeeding, atopic dermatitis, allergic rhinitis, asthma, and food hypersensitivity. The American Family Physician's Strength of Recommendation Taxonomy (SORT) was used to establish the quality of the studies and define the strength of recommendation.

Results: Three hundred and seven articles were obtained and seven met the inclusion criteria: one meta-analysis, one systematic review, two randomized clinical trials, one observational study, and two clinical guidelines. Overall, in relation to atopic dermatitis, it appears that breastfeeding is protective mainly up to two years of age, although some studies didn't find any association. In relation to allergic rhinitis, breastfeeding was protective up to five years of age and in asthma until 18 years of age. Finally, for food allergy, no association with breastfeeding was found.

Conclusions: Breastfeeding has a protective effect on the onset of asthma (SORT A) and allergic rhinitis (SORT B). Regarding food allergy and atopic dermatitis, the results are controversial, therefore, it is not possible to confirm its protective effect (SORT B). In face of this results further studies of good quality and for long periods of time are required.

Keywords: Breastfeeding allergic disease; Asthma; Allergic rhinitis; Atopic dermatitis. 